\title{
Importancia de la edad en la terapia de anticoagulación oral
}

\section{Dr. Claudio Parra Rocha}

Recibido el 17 de agosto de 2009, aceptado el 16 de diciembre de 2009

Rev Chil Cardiol 2009; 28: 395-396

\begin{abstract}
El tratamiento anticoagulante oral con antagonistas de la vitamina $\mathrm{K}$ para la prevención primaria o secundaria del tromboembolismo venoso o arterial, sigue siendo hasta el momento la mejor alternativa terapéutica teniendo en cuenta la relación beneficio vs. riesgo de su uso crónico, habitualmente a permanencia, o por largos períodos.

El objetivo de la terapia anticoagulante oral es eliminar o disminuir el número de eventos cardioembólicos en los pacientes con patologías que los predispongan a ellos. Para ello el nivel de anticoagulación expresado en INR, para una gran mayoría de los pacientes, se ha establecido como adecuado entre 2 y 3 , para una proporción menor de pacientes, hasta 3 y 4 y excepcionalmente en pacientes con muy alto riesgo de tromboembolismo arterial hasta 4.5.

El uso agudo y crónico de los antagonistas de la vitamina $\mathrm{K}$ conlleva un riesgo consubstancial de hemorragias que aumenta a medida de un aumento en el nivel de anticoagulación. A mayor INR mayor será el riesgo y la severidad de la hemorragia. Todo paciente que inicia terapia anticoagulante debe saber y aceptar este riesgo, debe saber que para disminuir el riesgo del tromboembolismo arteriovenoso se debe exponer a un riesgo aumentado de hemorragias.

Es el costo, entre otros, de esta terapia que no ha podido ser aún desplazada o reemplazada por otras terapias.

Con INR bajo 2 el riesgo de hemorragias intracerebrales ya no disminuye más, pero sin embargo el riesgo de tromboembolismo aumenta bastante. A medida de que el INR aumenta, disminuye el riesgo del tromboembolismo, pero sin un incremento significativo en la reducción de este riesgo con INR sobre 3 y si si comienza a aumentar el riesgo de las hemorragias, especialmente con INR sobre 3.5 - 4, y más. De tal manera, la mejor relación entre el beneficio (máxima prevención de tromboembolismo) y el riesgo (mínimo
\end{abstract}

de hemorragias) del uso de la terapia con antagonistas de la vitamina $K$ está en alcanzar y mantener un nivel de anticoagulación con un INR entre 2 y 3.

Lamentablemente en la práctica habitual, mantener un nivel de anticoagulación estable es tremendamente difícil y prácticamente imposible. En estudios bien controlados, en las cuales los controles y precauciones se extreman al máximo, sólo dos tercios de los INR están en el rango de 2 a 3 . Por otra parte, la eliminación del riesgo del tromboembolismo aún con INR en rangos óptimos no es de $100 \%$, y el paciente mantiene la posibilidad de tener eventos cardioembólicos, eso si con una menor incidencia, y afortunadamente también de menor tamaño, de menor severidad y con menos secuelas, es decir hay una protección cuantitativa y cualitativa.

El riesgo aumentado de hemorragia intracraneal debido a la terapia anticoagulante, la causa más frecuente de hemorragia mortal o incapacitante, es generalmente, en los grandes estudios de menos de $0.3 \% / a n ̃ o$. En los estudios observacionales y en la práctica clínica, que acumulan experiencia y eventos por muchos años, han demostrado que el riesgo de hemorragias es más alto en aquellos que ya han tenido un accidente vascular cerebral oclusivo previo y en los pacientes más viejos.

Los pacientes con fibrilación auricular no valvular tienen aumento del riesgo de tromboembolismo arterial, pero hay subgrupos con riesgo muy bajo y otros con riesgo muy alto, de tal manera de que si a todos por igual se le indicara el tratamiento anticoagulante, el riesgo de una hemorragia grave en el subgrupo de muy bajo riesgo de tromboembolismo, sobrepasa por mucho la probabilidad de los eventos oclusivos que se desean evitar. De esta forma hay un score con los factores de riesgo para ser aplicado en estos paciente y aumentar así el beneficio del tratamiento anticoagulante, es decir indicar el tratamiento anticoagulante en aquellos con mayor

Correspondencia: Dr. Claudio Parra Rocha

Cardiología Hospital del Salvador

Av. Salvador 364, Providencia - Santiago

Correo Eléctronico:cparrarocha@mi.cl 
riesgo y elegir otras alternativas en los con riesgo muy bajo. Los factores de riesgo agrupados en la sigla CHADS2 (en inglés), que contempla edad mayor de 75 años, presencia de Diabetes Mellitus, Insuficiencia Cardíaca, Hipertensión arterial y accidente vascular cerebral o crisis cerebral isquémica transitorias, se está usando para estratificar este riesgo e indicar el tratamiento anticoagulante. Cada factor de riesgo es un (1) punto y se adjudican dos (2) puntos cuando el factor de riesgo es el accidente cerebro vascular o la crisis cerebral isquémica transitoria. Este índice de riesgo CHADS2 puede ir de cero $(0)$ a seis (6) con una mayor frecuencia de eventos cardioembólicos a medida que aumenta el score CHADS2. Si el score CHADS2 es cero (0) el riesgo es mínimo y no se justifica dar terapia anticoagulante. Si el score CHADS2 es uno (1) puede elegirse según el paciente, la terapia anticoagulante o la terapia antiplaquetaria. Si el CHADS2 es mayor a uno (1) la indicación es la terapia anticoagulante.

La incidencia y la prevalencia de la Fibrilación auricular aumenta a medida de que aumenta la edad de las personas, por lo cual dado el aumento de las expectativas de vida en nuestro país, la población de más de 75 y de 80 años de edad está siendo cada vez más numerosa y activa, lo que ha hecho en la práctica que nos enfrentemos a una población más vieja, con mayor prevalencia de fibrilación auricular, con más riesgo de accidentes tromboembólicas invalidantes, pero también esta población de mayor edad, por muchas de sus peculiaridades, están en situación de tener mayor incidencia de complicaciones hemorrágicas graves y severas, incluso mortales con el uso de los antagonistas de la vitamina $\mathrm{K}$.

El trabajo que se publica en esta edición de la revista de la Sociedad Chilena de Cardiología y Cirugía Cardiovascular "La edad como factor determinante en la decisión para utilizar la terapia de anticoagulación oral: una auditoria de gestión clínica" de los Dr. Nigel, P. Murray y Dra. Esther Meroni L. ${ }^{1}$, pone énfasis en la edad como un elemento relevante a tener en cuenta a la hora de decidir la indicación de la terapia anticoagulante. Los pacientes de edad mayores (75 - 80 y más años) pueden presentar polipatologías, deterioros variables de sus órganos y sistemas, en especial cerebral, hepático, renal y digestivo, deterioro variable de su estado psicoemocional, cognitivo, uso permanente o por tiempo prolongado de varios medicamentos, tienen trastornos del equilibrio, con frecuencia con poca red de apoyo familiar, se les hace difícil el traslado a los centros médicos-hospitalarios. Estos y otros elementos propios de la edad avanzada hacen tremendamente difícil poder mantener un tratamiento anticoagulante con antagonistas de la vitamina $\mathrm{K}$, de manera estable en rangos de INR terapéuticos (2 a 3 ) y vemos que con mucha frecuencia experimentan variaciones bajo y sobre los valores adecuados de INR, colocándolos en situaciones de riesgo de complicaciones, en especial las hemorragias graves. Una situación preocupante es que estas variaciones de los INR, se producen aún en situaciones de pacientes de edad, con controles previos muy estables y sin mediar algún factor agudo detectable, por lo que hay que mantener presente de que hay situaciones y condiciones que ignoramos y que se pueden manifestar en estas variaciones significativas de los INR de manera impensada. En este contexto, tiene mucho valor lo que se señala en el trabajo de tener programas de educación a estos pacientes, instruirlos, y apoyarlos con clínicas o laboratorios o centros de tratamiento anticoagulantes, donde se concentre la experiencia de médicos, tecnólogos, enfermeras y técnicos paramédicos en la elaboración de los esquemas, control de estos pacientes, actividades de prevención, detección y tratamiento precoz de las complicaciones, atención permanente en horarios hábiles para solucionar las dudas y problemas de los pacientes y tener registro de todas las complicaciones y el nivel de cumplimiento de los INR terapéuticos programados. Creo que en situaciones de duda, tiene, y mucho valor el objetivar el nivel físico-cognitivo de los pacientes de edad mediante test simples que ayuden a definir el grado de entendimiento y la capacidad de adherencia a esta terapia que es altamente efectiva en disminuir los riesgos del tromboembolismo arterial pero que si no es llevada de manera adecuada es también altamente peligrosa por las complicaciones hemorrágicas que puede determinar.

\section{Referencias}

1.- Dr. Nigel P. Murray y Dra. Esther Meroni L. y cols. La edad como factor determinante en la decisión para utilizar la terapia de anticoagulación oral: una auditoria de gestión clínica. 